\title{
Active Shape Model-Based Segmentation of Digital X-ray Images
}

\author{
G. Behiels ${ }^{1}$, D. Vandermeulen ${ }^{1}$, F. Maes ${ }^{1}$, P. Suetens ${ }^{1}$, and P. Dewaele ${ }^{2}$ \\ 1 Medical Image Computing, Katholieke Universiteit Leuven, Leuven, Belgium \\ 2 Agfa-Gevaert NV, Mortsel, Belgium
}

\begin{abstract}
We propose an improved search procedure for Active Shape Model (ASM) based delineation of anatomical structures in digital X-ray images. Whereas the original ASM search method [1] iteratively improves the current estimate of the location of boundary points by a limited least squares adjustment of the pose and shape parameters, our method additionally requires the subsequent changes in shape during the search to be smooth, which is achieved by using a minimum cost path search algorithm. We compare the two methods on a database of more than 400 manual segmentations of digital X-ray images of the femur, humerus and calcaneus. We evaluate the accuracy and robustness of both methods using a cross-validation procedure.
\end{abstract}

\section{Introduction}

Accurate detection and segmentation of anatomical structures is an essential component in many biomedical image analysis procedures. Delineation of bone structures, for instance in digital X-ray (DRX) images, is a prerequisite in many orthopaedic examinations. Since manual delineation of these anatomical structures is very tedious and time consuming, fast and accurate computer-aided segmentation methods are required.

Cootes et al. [1] represent objects as sets of labeled points and examine the statistics of their coordinates over a number of training examples. The characteristic pattern of a shape class is described by the average shape vector and a linear combination of eigenvectors of the variations around the average shape. These representations are called Point Distribution Models (PDM). During image search, new target points, e.g. edges, are searched in a region of the image around each model point and the model is updated to best fit these new target points. This update consists of two steps. First, the pose parameters (rotation, translation, scaling) are determined, and second, the remaining residuals are projected onto the eigenvector basis. The resulting shape parameters are limited to ensure that the current shape remains similar to the training set. In the context of locating object boundaries in images using an iterative search algorithm to fit the statistical object model to the image data, Point Distribution Models are coined Active Shape Models (ASM).

A shortcoming of this search method, however, is the independent estimation of each point to a new target position by a local search along the normal direction 
toward the strongest image edges. Inaccuracies in this estimation, e.g. edges corresponding to a different object, cannot be accommodated for appropriately by the pose and shape parameters, even when using a different criterion for positioning new target points like maximum similarity of grey level profiles [2].

We therefore propose to estimate the position of new target points while incorporating a regularisation term imposing smoothness of shape changes. New target point positions are determined using a minimum cost path search algorithm before the adjustment of pose and shape parameters.

\section{Point Distribution Model: Capturing Shape Statistics}

Point Distribution Models represent each object boundary as a vector $s$ of boundary point coordinates: $s=\left(\begin{array}{lllllll}x_{0} & y_{0} & x_{1} & y_{1} & \ldots & x_{n} & y_{n}\end{array}\right)^{T}$. These boundary points correspond to expert-labeled landmark points on examples of objects belonging to the same shape class. To eliminate changes in scale, rotation and translation, shapes in the training set are aligned by minimizing a weighted sum of squared distances between corresponding points on the different examples of the training set for a single shape class [4]. The average or mean shape $\bar{s}$ and the covariance matrix $C_{\mathrm{tr}}$ of variations about the mean are computed. The eigenvectors $p_{k}$ of the covariance matrix represent an orthogonal basis of linear deformation modes that describe how landmark points tend to move together as the shape varies. The eigenvalue $\lambda_{k}$ associated to each eigenvector $p_{k}$ is equal to the variance explained by each linear deformation mode. Each shape $s$ of the training set can then be represented as a weighted sum of the eigenvectors $p_{k}$ and the mean $\bar{s}$, $s=\bar{s}+P b$, wherein $P=\left(\begin{array}{llll}p_{1} & p_{2} & \ldots & p_{2 n}\end{array}\right)$ is a matrix with the eigenvectors as its columns, and $b=\left(\begin{array}{llll}b_{1} & b_{2} & \ldots & b_{2 n}\end{array}\right)$ the vector of weights or the shape coefficients of the shape in our the model-space $(\bar{s}, P)$. Suitable limits for the shape parameters are, for example, $-3 \sqrt{\lambda_{k}} \leq b_{k} \leq 3 \sqrt{\lambda_{k}}$. Because most of the variance is explained by the eigenvectors with the largest eigenvalue, the number of eigenvectors can be limited to $t<2 n$, defined e.g. as the smallest number of modes such that the sum $\sum_{i=1}^{t} \lambda_{i}$ of variance explained, is a sufficiently large proportion of the total variance, $\lambda_{\mathrm{T}}=\sum_{i=1}^{2 n} \lambda_{i}$. In our experiments we choose $t$ to be the smallest number satisfying the following condition

$$
\frac{\sum_{i=1}^{t} \lambda_{i}}{\lambda_{\mathrm{T}}}>0.9
$$

thus explaining more than $90 \%$ of the variance of the training set.

Each PDM for a particular shape class is built using a bootstrap-procedure. The first image is presented to a trained expert who carefully delineates the object. For the next three images, the first delineation can be interactively transformed and the shape boundary points are manually adjusted to the correct object boundary. After these four manual segmentations, a shape model is computed and used to automatically segment the other images. Each presented solution can be edited before it is in turn added to the shape model. 


\section{Image Model: Capturing Image Appearance}

Given a shape model and an image containing an example of the modelled shape class, detection or segmentation of the object involves searching for the model parameters that best fit the model to the image. The image model defines which image feature and/or which similarity function to use for measuring the quality of the fit of the model to the image data. During optimization, new target point positions that best fit the image model are searched for in a local neighborhood. In the original ASM implementation, these points were defined to have the highest image gradient magnitude within a search window, along a line normal to the current estimate of the contour.

Similarly to [2] where different image models on photographic images of a human face are compared, we observed that searching for the highest gradient point is not the most reliable measure to define the boundary. We performed an extensive test on a database of over 400 digital X-rays of 6 different bone structures such as the femur, humerus, etc. By way of example we show the results pictorially for the femur. In Fig. 1, one image of the training set is shown together with the manual delineation as a solid line. For each of the 104 examples of femur images, we virtually displaced each contour point to the point with the largest gradient magnitude within a local search interval of 20 pixels along the normal to the contour. The mean displacement over all images for each contour point is displayed as a circle in Fig. 1 together with the corresponding standard deviation as a bar through these circles. We observe that there is a strong bias and variance for those contour points in areas of weak contrast and vice versa for contour points on strong edges.

Alternative image appearance models define a similarity measure on image feature values along one-dimensional profiles normal to the contour. Similarly to the boundary point coordinates, these profiles can be modelled during a learning phase as follows. Each profile $g$ of the training set can be represented by the mean profile $\bar{g}$ and a number of modes of variation $P_{g}$

$$
g=\bar{g}+P_{g} b_{g}
$$

A suitable similarity measure defining the fit of a new input profile $x$ with $n_{p}$ points to the model profile is given by the Mahalanobis distance $M$

$$
M=\sum_{j=1}^{n_{p}} \frac{b_{g j}}{\lambda_{j}}
$$

wherein $b_{g j}$ is the projection of the input pattern $x$ onto the $j$-the eigenvector $p_{g j}$. If we only use a limited number of eigenvectors $t_{g}$, Equation (1) can be approximated by (see [2])

$$
F=\sum_{j=1}^{t_{g}} \frac{b_{g j}^{2}}{\lambda_{j}}+\frac{2 \epsilon^{2}}{\lambda_{t_{g}}}
$$

wherein $\epsilon^{2}$ is the sum of squares of differences between the input pattern $x$ and the reconstruction of this pattern $\bar{g}+P_{g} b_{g}$. 
We compared four different image features $g$ (intensity, normalized intensity, gradient, normalized gradient) on the database. We graphically show the results for the femur in Fig. 1 and summarize the results for the other structures in Table 1. From these experiments, we conclude that normalized intensities are the better image features in combination with the Mahalanobis distance similarity measure.
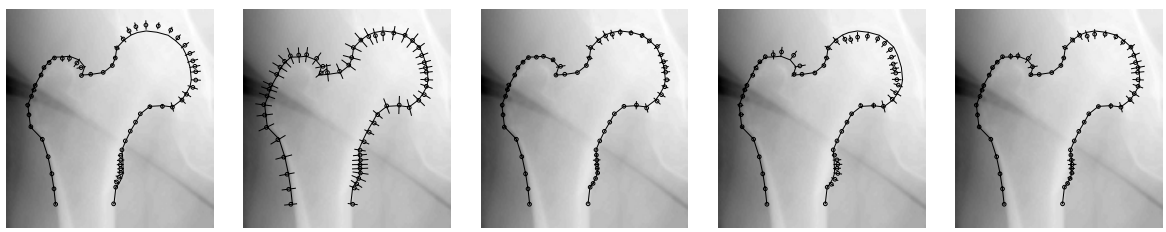

Fig. 1. Mean error (circle) and standard deviation (bars) for a local search along the normals using the gradient-magnitude or (2). The first image displays the results for a local search to the image points with the largest magnitude of the gradient. The second image displays the results for intensity profiles, the third represents normalized intensity and the fourth and fifth respectively gradient and normalized gradient profiles.

Table 1. Mean error and standard deviation when searching for the maximum gradient $(\Delta)$ and for profile matching using intensity $(I)$, normalized intensity $(\tilde{I})$, gradient $(G)$ and normalized gradient $(\tilde{G})$ profiles.

\begin{tabular}{rccccc}
\hline & $\Delta$ & $I$ & $\tilde{I}$ & $G$ & $\tilde{G}$ \\
\hline \hline calcaneus & $1.81 \pm 2.59$ & $5.73 \pm 6.96$ & $1.46 \pm 2.36$ & $1.95 \pm 2.87$ & $1.61 \pm 2.50$ \\
femur(de,lv) & $1.74 \pm 2.73$ & $5.56 \pm 6.67$ & $1.26 \pm 2.14$ & $1.77 \pm 2.88$ & $1.46 \pm 2.30$ \\
femur(pe,vv) & $2.19 \pm 2.63$ & $6.14 \pm 7.24$ & $1.20 \pm 2.16$ & $2.48 \pm 2.96$ & $1.96 \pm 2.68$ \\
femur(de,vv) & $1.54 \pm 2.46$ & $5.91 \pm 7.01$ & $1.17 \pm 1.94$ & $1.56 \pm 2.69$ & $1.17 \pm 2.10$ \\
tibia & $2.01 \pm 2.75$ & $5.11 \pm 6.25$ & $1.30 \pm 1.98$ & $2.25 \pm 2.91$ & $1.79 \pm 2.55$ \\
humerus & $0.99 \pm 1.83$ & $5.35 \pm 7.71$ & $0.89 \pm 1.74$ & $1.16 \pm 2.00$ & $0.99 \pm 1.75$ \\
\hline
\end{tabular}

\section{Search Algorithms}

Given the statistical shape and image appearance models for a particular structure in a particular imaging modality, our aim is to use them to segment these structures into new images by searching for the shape parameters $b=\left(b_{1} b_{2} \ldots b_{t}\right)^{T}$ and pose parameters (scale $s$, rotation $\theta$ and translation $t_{x}$ and $t_{y}$ ) that best fit the model to the data. In Sect. 4.1, we explain the original search algorithm used by Cootes et al. [3] and in Sect. 4.2 we explain the modifications we propose to constrain the search procedure.

\subsection{Iterative Search}

Given an initial estimate of the pose and shape parameters of an instance of the object in the image, a region of the image around each boundary point is 
examined to determine a displacement required to move that point to a better image position. In our implementation we search along the normal to the curve for that point with the smallest Mahalanobis distance (or its approximation given by (2)) between the model profile and the image feature profile centered at that point. The pose and shape parameters are then adjusted to move the boundary points as close as possible to the suggested target points, while still satisfying the model shape constraints. The original method suggested by Cootes et al. [2] first calculates the pose parameters (scale, translation, rotation) that best fit (in a least squares sense) the current estimate $X$ to the suggested set of points $X+d X$. The residual displacements $d x$ (expressed in the local model coordinate frame) are then approximated (projected onto the ASM eigenvectors) by the incremental changes in the shape parameters $d b=P_{t}^{T} d x$ with $P_{t}$ the sub-matrix of $P$ containing only the first $t$ columns of $P$. This procedure is repeated until no significant changes result.

\subsection{Regularized Iterative Search Using Dynamic Programming}

At each update step in the previous search algorithm, pose and shape parameters are adjusted using a least squares approximation procedure. This least squares fit criterion is known to be sensitive to outliers occasionally suggested by the imperfect image model. (Fig. 2). Indeed, suppose we start from an initialized shape model $X$ and take the target points with the smallest Mahalanobis distance, we might end up with a shape like the dotted line in Fig. 2. Here, model point $p_{2}$ is attracted to an outlier position. Adjusting the shape and pose parameters then leads to a biased boundary estimate that cannot be corrected during further iterations. We propose to correct the suggested movements $d X$ before adjusting pose and shape parameters in the following way.
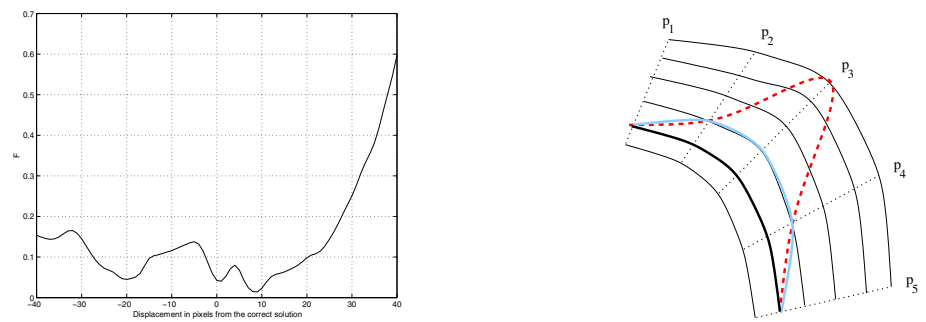

Fig. 2. Left:Mahalanobis distance calculated using (2) with normalized intensity profiles. The values are computed for a given boundary point along a path normal to the curve. Right:Starting from an initialized shape model (bold line), displacements to target points $p_{i}$ with the smallest Mahalanobis distance can result in an outlier configuration (dashed line). Instead, a regularized target point configuration (grey line) is more appropriate. 
We construct a "cost-matrix" $K$ with dimensions $n \times l$ ( $n$ being the number of boundary points), containing the associated Mahalanobis distance values along the one-dimensional search profiles (length $l$ pixels) normal to the curve. We then incorporate a regularization constraint penalizing outlier configurations by minimizing the function $f$

$$
f(X)=K_{1, x_{1}}+\sum_{i=2}^{n}\left(K_{i, x_{i}}+\alpha\left|x_{i}-x_{i-1}\right|\right)
$$

wherein $\alpha$ is a weight factor. We minimize $f$ for $X$ where $X$ are the displacements in pixels along the normal to the curve: $X=\left(x_{1} x_{2} \ldots x_{n}\right),-\frac{l}{2} \leq x_{i} \leq \frac{l}{2}$. The last term in (3) indeed penalizes local outlier positions. This function can be minimized using dynamic programming. The corrected displacements are then used for updating the shape and pose parameters as in the previous search procedure.

\section{Results}

In this section we describe experiments designed (1) to test how well Point Distribution Models can model manual delineations of bone structures, (2) to test how well the image model can model the expected appearances of these structures in DRX, (3) to compare the results of the original iterative ASM search procedure and the proposed improved MCP search procedure.

These tests are based on a cross-validation or jack-knifing (leave-one-out) procedure. For each training set of a particular anatomical structure, we iteratively leave one example out and compute a shape and image model based on the remaining examples. This model is then used to segment the one image left out, and the average and maximal correspondence error and boundary error between the solution and the manual segmentation are computed. The correspondence error $E_{c}$ of each point is the distance of the point to its corresponding point of the manual segmentation. The average and maximal error of the correspondence (over all boundary points of all examples) are denoted by $E_{c a}$ and $E_{c m}$, respectively. The boundary error $E_{b}$ of a point is the distance between this point and the closest boundary point of a spline computed through the manual segmentation. The average and maximal boundary error are denoted by $E_{b a} \leq E_{c a}$ and $E_{b m} \leq E_{c m}$.

Each test is performed on a database of more than 400 segmentations with the following subdivision: femur 104 (proximal end, ventral view), humerus 65 (proximal end, ventral view), calcaneus 91 (lateral view), femur 92 (distal end, lateral view), femur 56 (distal end, ventral view), tibia 17 (proximal end, ventral view).

The first experiment is designed to test how well the contours drawn manually by an expert can be modelled by the Point Distribution Models. Since we iteratively left one example out of the training set and since we also use a limited number of eigenvectors and limit the shape parameters between $-3 \sqrt{\lambda_{k}} \leq b_{k} \leq$ $3 \sqrt{\lambda_{k}}$, we cannot expect the PDM based on the remaining examples to model 
the one example perfectly. The average as well as maximal correspondence and boundary errors are given in Table 2 . These values represent lower limits on the errors obtained in the other experiments.

Table 2. Error measure for modelling the shape of the objects: $E_{c a}$ is the average correspondence error; $E_{b a}$ is the average boundary error; $E_{c m}$ is the maximum correspondence error. $E_{b m}$ is the maximum boundary error.

\begin{tabular}{rrccc}
\hline & $E_{c a}$ & $E_{b a}$ & $E_{c m}$ & $E_{b m}$ \\
\hline \hline calcaneus & 1.93 & 1.32 & 12.49 & 8.20 \\
femur (de,lv) & 1.51 & 1.13 & 12.79 & 9.35 \\
femur (pe,vv) & 1.55 & 1.00 & 9.78 & 6.79 \\
femur (de,vv) & 1.83 & 1.41 & 8.28 & 7.10 \\
tibia & 2.14 & 1.43 & 8.80 & 6.46 \\
humerus & 1.08 & 0.82 & 7.04 & 4.21 \\
\hline
\end{tabular}

In the second test we compare both search algorithms, the iterative search algorithm (ASM)and the Minimal Cost Path-based (MCP) search algorithm, with respect to their capability of finding the object boundary if the initial position is optimal w.r.t. the shape model (the solutions of the first test are given as initial positions). In both cases we use as image-model fit measure the Mahalanobis distance on the normalized grey-level profiles. We stop the search algorithm after 1 iteration and compute the errors (see Table 3). These errors reflect the stability of both search algorithms around the optimal shape. Corresponding errors after convergence are also listed in Table 3.

Table 3. Error measure for stability of the search algorithm near the correct solution. The values given in the left section of the table are the average correspondence $\left(E_{c a}\right)$ and boundary $\left(E_{b a}\right)$ errors, and maximal correspondence $\left(E_{c m}\right)$ and boundary $\left(E_{b m}\right)$ errors for both the original ASM search method and the improved MCP method after one iteration. The values in the right section of the table are the errors compiled after convergence of the algorithm.

\begin{tabular}{rl|rrrr||rrrr}
\hline & & $E_{c a}$ & $E_{b a}$ & $E_{c m}$ & $E_{b m}$ & $E_{c a}$ & $E_{b a}$ & $E_{c m}$ & $E_{b m}$ \\
\hline \hline calcaneus & Asm & 2.45 & 1.65 & 21.48 & 14.09 & 5.89 & 2.56 & 46.24 & 26.13 \\
& Mcp & 2.16 & 1.58 & 19.61 & 12.48 & 2.61 & 1.53 & 21.49 & 21.09 \\
\hline femur (de,lv) & Asm & 2.15 & 1.69 & 14.81 & 11.58 & 3.87 & 2.33 & 31.20 & 31.00 \\
& Mcp & 1.95 & 1.51 & 14.56 & 11.79 & 2.68 & 1.78 & 32.05 & 27.85 \\
\hline femur (pe,vv) & Asm & 1.90 & 1.26 & 11.16 & 7.24 & 3.25 & 1.76 & 29.84 & 20.75 \\
& Mcp & 1.69 & 1.02 & 10.77 & 7.94 & 1.91 & 1.03 & 13.80 & 9.63 \\
\hline femur (de,vv) & Asm & 2.41 & 1.81 & 13.10 & 12.28 & 5.33 & 3.19 & 60.74 & 52.51 \\
& Mcp & 2.02 & 1.45 & 10.56 & 10.00 & 2.46 & 1.57 & 18.27 & 16.34 \\
\hline tibia & Asm & 2.51 & 1.70 & 15.45 & 12.54 & 4.51 & 2.65 & 24.37 & 18.43 \\
& Mcp & 2.32 & 1.49 & 12.35 & 7.33 & 2.78 & 1.61 & 17.83 & 12.93 \\
\hline humerus & Asm & 1.53 & 1.18 & 9.31 & 8.90 & 2.77 & 1.75 & 22.88 & 21.58 \\
& Mcp & 1.23 & 0.91 & 7.40 & 6.38 & 1.36 & 0.93 & 8.85 & 8.39 \\
\hline
\end{tabular}


The third test compares the accuracy of both algorithms when starting from the registered mean shape with each manual segmentation. This experiment evaluates the stability of both search methods given that the pose parameters are already optimal. The results of this test are found in Table 4. Examples of segmentations with the worst correspondence are given in Fig. 3.
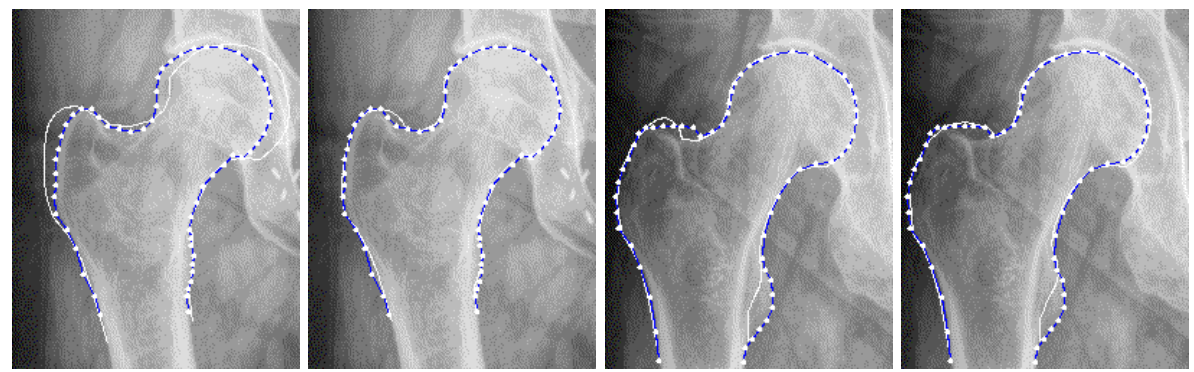

Fig. 3. Segmentation of the femur with the worst correspondence error (ASM: first, MCP: last image) computed after registering the average shape model with the manual segmentation. Corresponding segmentation obtained with the other method (MCP: second, ASM: third image). The manual segmentation is given by the dotted line.

Table 4. Error measures for segmentation after registering the mean shape with the manual segmentation using the ASM and MCP search technique.

\begin{tabular}{cc|rrrr}
\hline & & $E_{c a}$ & $E_{b a}$ & $E_{c m}$ & $E_{b m}$ \\
\hline \hline calcaneus & Asm & 7.61 & 2.96 & 64.95 & 44.96 \\
& Mcp & 4.10 & 1.70 & 39.45 & 24.35 \\
\hline femur (de,lv) & Asm & 5.84 & 2.72 & 46.34 & 28.28 \\
& Mcp & 4.19 & 2.27 & 27.84 & 26.25 \\
\hline femur (pe,vv) & Asm & 4.95 & 2.43 & 36.71 & 36.71 \\
& Mcp & 3.10 & 1.34 & 23.75 & 18.03 \\
\hline femur (de,vv) & Asm & 7.66 & 4.47 & 68.07 & 50.15 \\
& Mcp & 3.49 & 1.87 & 24.73 & 21.08 \\
\hline tibia & Asm & 5.15 & 2.99 & 35.73 & 31.44 \\
& Mcp & 3.33 & 1.75 & 17.92 & 13.42 \\
\hline humerus & Asm & 4.09 & 2.12 & 28.18 & 22.63 \\
& Mcp & 2.24 & 1.09 & 18.05 & 14.27 \\
\hline
\end{tabular}

The last experiment examines the robustness of both search algorithms w.r.t. changes in the initial pose parameters. After registration of the mean shape with the manual segmentation, one of the pose parameters is varied and the model is 
fitted to the image. To make the search-algorithms more robust, we adaptively increase the number of eigenvectors for the shape during the search. In Table 4 the average correspondence errors for both search algorithms are displayed.
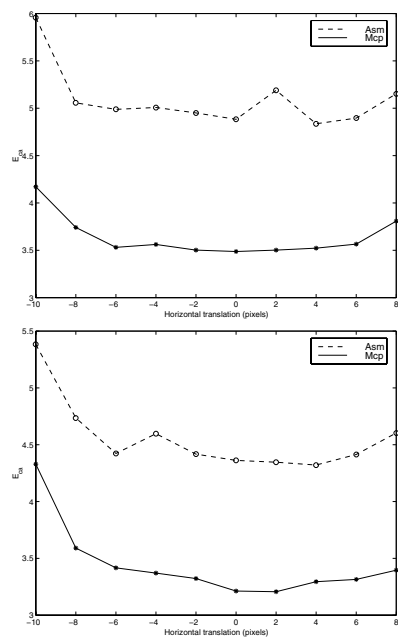
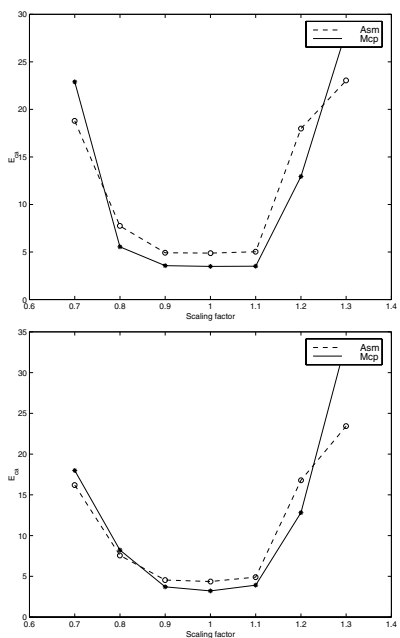
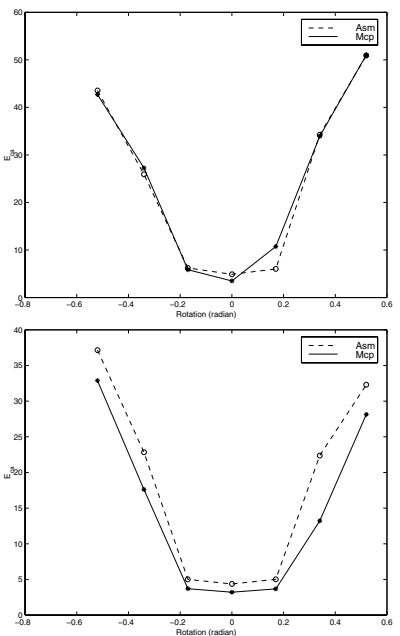

Fig. 4. Sensitivity to initial parameters experiment for the tibia (first row ) and the femur (pe,vv,second row). These values are computed using a scheme which increases the number of eigenvectors used during the search.

To evaluate the speed of both search algorithms, we measured the total calculation time on a search of a shape containing 25 points over 56 images after registration of the mean with the manual segmentation. The test was done on an Intel Pentium 300MHZ processor using Linux. The average calculation times till convergence for one shape are $0.2 \mathrm{~s}$ for the original search method and $0.18 \mathrm{~s}$ for the MCP-algorithm.

\section{Discussion}

We compared the original ASM iterative search method to the regularized MCP search method using a cross-validation procedure with expert manual segmentations as the standard. Tables 1 and 2 represent independent lower limits on the obtainable accuracy because of limitations in the shape model and limitations and imperfections in the image model resp. which prevents both search methods from perfectly recovering the manual segmentations.

Comparing the results of the stability test (one iteration starting from the shape fitted to the manual segmentation) of both algorithms (Table 3) to these lower limits indicates that the one iteration of the MCP method consistently outperforms the ASM method but still occasionally fails to correct for outliers suggested by the image fit model. These errors are not recovered during remaining iterations as shown in Table 3. 
When starting from the mean shape after registration with the manual segmentation, the MCP method again performs better according to all error measures (Table 4). Figure 3 shows two examples of worst results for both algorithms as well as the corresponding solution by the other algorithm. From this figure we observe that ASM worst case delineations tend to be associated more with gross overall misalignments. This can be attributed to the fact that outliers in ASM are inevitably propagated to pose and gross shape parameters, whereas the MCP algorithm regularizes these outliers and generates delineations with only local deviations.

Figure 4 illustrates that both search algorithms are relatively robust for initial mismatches in model translation, but degrade abruptly for slight changes in both scale and rotation. Overall the MCP algorithm performs more robustly. However, in general, we can conclude that both automatic delineation methods require that the initial pose parameters (translation, rotation, scaling) be fairly close to their final values. This sensitivity to mismatches of the initial model position can be reduced to some extent by using global optimization search algorithms such as Genetic Algorithm search [6] or by a multi-resolution search (which includes a multi-resolution shape and image model as well) [5].

Despite the added algorithmic complexity of the minimum cost path search algorithm, the MCP algorithm is still, but only marginally, faster than the original ASM algorithm. Each iteration of the MCP algorithm indeed takes more time than the ASM algorithm, but far less iterations are needed for convergence.

\section{Conclusions}

This work presents a new search technique for delineation of anatomical structures in DRX using statistical Point Distribution Models. The image-model fit function that performs best for these examples is shown to be the Mahalanobis distance similarity function on normalized grey-level profiles. In order to minimize the sensitivity of the original iterative search procedure [1] to outliers, we incorporate a regularization constraint penalizing outlier configurations that we minimize using a dynamic programming algorithm. Extensive cross-validation experiments comparing both search procedures indicate that although the search procedure proposed in this paper consistently outperforms the original procedure for similar execution times, it still occasionally diverges to incorrect solutions because of limitations in both the shape and image model. Current work focuses on improving both components and rephrasing the procedure by optimizing a Bayesian Maximum a Posteriori (MAP) objective function [7].

\section{Acknowledgements}

This work was supported by a grant of Agfa-Gevaert NV, by a grant for research specialization from the Flemish Institute for stimulation of the scientifictechnological research in the industry (IWT), and by the Research Fund KU Leuven GOA/99/05 (Variability in Human Shape and Speech). The manual segmentations were performed by Dr. Tom De Jaegere. 


\section{References}

1. T. F. Cootes and C. J. Taylor. Active shape models - "smart snakes". In BMVC, pages 256-275, 1992. 128, 137

2. T. F. Cootes and C. J. Taylor. Active shape model search using local grey-level models: A quantitative evaluation. In $B M V C$, pages 339-348, Sept. 1993. 129, 130, 132

3. T. F. Cootes, C. J. Taylor, D. H. Cooper, and J. Graham. Training models of shape from sets of examples. In BMVC, pages 9-18, 1992. 131

4. T. F. Cootes, C. J. Taylor, D. H. Cooper, and J. Graham. Active shape models - their training and application. Computer Vision and Image Understanding, 61(1):38-59, January 1995. 129

5. T. F. Cootes, C. J. Taylor, and A. Lanitis. Active shape models : Evaluation of a multi-resolution method for improving image search. In $B M V C$, pages 327-336, 1994. 137

6. A. Hill, C. J. Taylor, and T. Cootes. Object recognition by flexible template matching using genetic algorithms. In 2nd European Conference on Computer Vision, pages 852-856, May 1992. 137

7. Y. Wang and L. H. Staib. Boundary finding with correspondence using statistical shape models. In Proc. IEEE Conf. Computer Vision and Pattern Recognition, pages 338-345, 1998. 137 